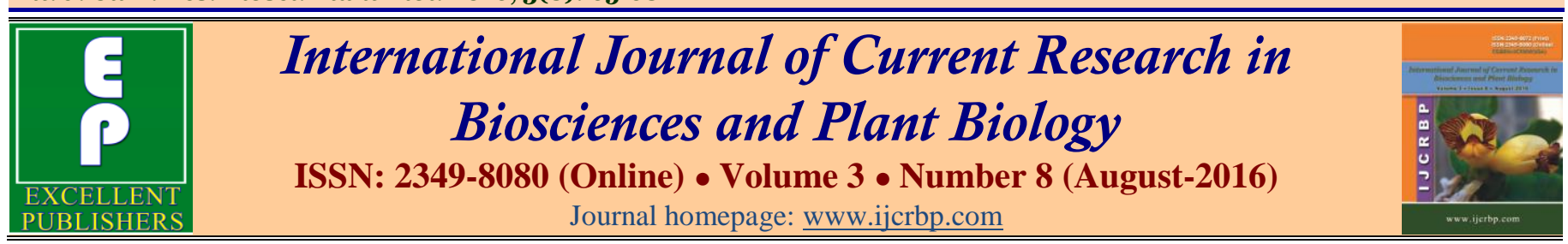

\title{
Adult Diabetic Ketoacidosis: Precipitating Factors, Diagnostic Criteria and Management Strategies at Elobied Hospital - West Sudan
}

\author{
AbdlelSalam Mohamed Hamad Elfaki ${ }^{*}$ and Mohamed Ahmed Agab² \\ 1\& 2MD-Department of Internal Medicine, Faculty of Medicine and Health Sciences - University of Kordofan, Sudan \\ *Corresponding author.
}

\section{A bstract}

Diabetic ketoacidosis (DKA) is the most common hyperglycaemic emergency in patients with diabetes mellitus. This study aimed to determine the precipitating factors, diagnostic criteria and management strategies of adult diabetic ketoacidosis at Elobied Hospital. In this hospital-based retrospective study, the records of 53 patients who were admitted to medical wards in period from January 2015 to December 2015 were reviewed. The records of 53 patients admitted to the medical wards with diabetic ketacidosis were reviewed. Sixteen $(30 \%)$ were females while $37(70 \%)$ were males. The common presenting symptoms were polyuria (57\%), polydypsia (55\%), nausea and vomiting (30\%), fever (30\%), and epigastric pain (19\%). Infections were the leading precipitating factors for DKA (47\%). Malaria constituted almost half of these infections (48\%). Interrupted insulin therapy was the second common precipitating factor for DKA reported in $28 \%$ of patients (noncompliance). Seventeen (32\%) of patients were newly diagnosed diabetics and presented with DKA. The diagnosis of DKA was made depending on the presenting symptoms, hyperglycaemia, and the presence of glucose and acetone in the urine. Mean blood glucose level in patients with DKA was $368 \pm 72$ $\mathrm{mg} / \mathrm{dl}$. In this study all patients admitted with DKA were managed by giving soluble insulin for hyperglycaemia and $0.9 \% \mathrm{NaCl}$ for correction of fluid deficit beside treatment of infections if present. Records showed no evidence of bicarbonate or potassium administration. Since the majority of cases of DKA occurs in patients who are known diabetics, this complication should be largely preventable by education of patients, health care professionals and the general public. Laboratory facilities at Elobied hospital must be improved to encourage early proper diagnosis of DKA and determine the severity of DKA to decide where the patient can be managed, in the intensive care unit or in the general medical wards. Intensive care unit (ICU) must be established to provide good care for severe DKA and other emergency cases.
\end{abstract}

Article Info

Accepted: 22 July 2016

Available Online: 06 August 2016

Ke ywords

Adult diabetic ketoacidosis

Diabetes mellitus

Hyperglycaemia

Metabolic acidosis

\section{Introduction}

Diabetic emergencies associated with diabetic ketoacidosis (DKA) and a hyperglycemic hyperosmolar state (HHS), both are acute life-threatening metabolic disturbances. DKA is the most common hyperglycaemic emergency in patients with diabetes mellitus and it is most often occurs in patients with type1 diabetes mellitus, but patients with type 2 diabetes are susceptible to DKA under stressful conditions such as trauma, surgery, or infection. The basic underlying mechanism for diabetic ketoacidosis is insulin deficiency coupled with elevated levels of counter-regulatory hormones, such as glucagon, cortisol, catecholamines, and growth 
hormone. DKA is characterized by hyperglycaemia, acidosis, dehydration, and electrolytes abnormalities, which result from the above mentioned basic underlying mechanism. The classical clinical picture of patients with DKA includes a history of polyuria, polydypsia, weight loss, vomiting, abdominal pain, dehydration, weakness, mental status change and coma. Physical finding include poor skin turgor, Kussmaul respiration, tachycardia, hypotension, alteration in mental status, shock, and ultimately coma. The main precipitating factors of DKA are infections and missed insulin injection. Since 20-30\% of patients presenting in DKA do so as the initial manifestation of their previously undiagnosed disease, physician must maintain a high level of suspicion for this condition (Umpierrez et al., 2002; Nugent, 2005).

Elobeid is the capital of North Kordofan state. Elobeid Teaching Hospital is a teaching hospital with a wide catch up area including different states (North Kordofan, West Kordofan, South Kordofan, and parts of Darfour states). This study aimed to determine the precipitating factors, diagnostic criteria and management strategies of adult diabetic ketoacidosis at Elobied Hospital.

\section{Methods}

In this hospital-based retrospective study, the records of 53 patients who were admitted to medical wards in period from January 2015 to December 2015 were reviewed. A data sheet was designed for collecting: the socioeconomic data, duration of diabetes, drugs used for treatment diabetes mellitus, presenting symptoms, precipitating factors, investigations which were done, and medications used in treatment of DKA. Data were analyzed using Statistical Package for Social Sciences (SPSS) version 18.

\section{Results}

The records of 53 patients admitted to the medical wards with diabetic ketoacidosis were reviewed. Sixteen (30\%) were females while $37(70 \%)$ were males. The common presenting symptoms were polyuria (57\%), polydypsia (55\%), nausea and vomiting (30\%), fever (30\%), and epigastric pain (19\%) as shown in Table 1.

Table 1. Common presenting symptoms of the patients admitted in medical wards Elobeid Teaching Hospital.

\begin{tabular}{lll}
\hline Symptom & Frequency & Percent \% \\
\hline Polyuria & 29 & $55 \%$ \\
Polydypsia & 30 & $57 \%$ \\
Nausea and vomiting & 16 & $30 \%$ \\
Fever & 16 & $30 \%$ \\
General fatigue & 15 & $28 \%$ \\
Epigastric abdominal pain & 10 & $19 \%$ \\
\hline
\end{tabular}

The elicited physical signs include dehydration, hypotension, sinus tachycardia, and alteration in mental status. Infections were the leading precipitating factors for DKA (47\%). Malaria constituted almost half of these infections $(48 \%)$. Interrupted insulin therapy was the second common precipitating factor for DKA constituting $28 \%$ (non-compliance) (Table 2).

Table 2. Precipitating factors for DKA.

\begin{tabular}{lll}
\hline Precipitating factor & Frequency & Percent \% \\
\hline Infections & 25 & $47 \%$ \\
Interrupted insulin therapy & 15 & $28 \%$ \\
No recognized precipitating factor & 13 & $25 \%$ \\
\hline
\end{tabular}

Table 3. Systolic blood pressure in various age groups

\begin{tabular}{llll}
\hline \multirow{2}{*}{ Age groups } & Systolic blood pressure & & Total \\
\cline { 2 - 3 } & $\mathbf{> 9 0} \mathbf{~ m m H g}$ & $<\mathbf{9 0} \mathbf{~ m m H g}$ & 10 \\
15- 25 years & 10 & 0 & 07 \\
26-35 years & 07 & 0 & 12 \\
36-45 years & 12 & 0 & 11 \\
46- 55 years & 11 & 0 & 13 \\
$>$ 55 years & 06 & 7 & $\mathbf{5 3}$ \\
Total & $\mathbf{4 6}$ & $\mathbf{0 7}$ & \\
\hline
\end{tabular}


Seventeen (32\%) of patients were newly diagnosed diabetics and presented with DKA. Mean blood glucose level in patients with DKA was $368 \pm 72 \mathrm{mg} / \mathrm{dl}$. Fourteen (26\%) patients were diagnosed as severe DKA due to presence of systolic hypotension (systolic blood pressure $<90 \mathrm{mmHg}$ ) and/or tachycardia (Heart rate > 100 beat/minute). All patients with severe DKA belonged to elder age group (> 55 years) (Table 3 ).

\section{Discussion}

The discovery of insulin in 1921 changed the life expectancy of patients with diabetes mellitus dramatically. Today, almost a century later, diabetic ketoacidosis (DKA) and hyperglycaemic hyperosmolar state (HHS) remain significant causes of morbidity and mortality across different counties, ages, races, and socioeconomic groups and a significant economic burden for society (Maletkovic and Drexler, 2013). In this study infections were the major precipitating factors for DKA occurring in $47 \%$ of patients, with malaria constituted $48 \%$ of these infections. Similar percent of infection was reported by Umpierrez and Kitabchi but the difference was that in their study urinary tract infection and pneumonia account for the majority of infections (Umpierrez and Kitabchi, 2003).

Diagnostic criteria for DKA include presence of blood glucose more than $250 \mathrm{mg} / \mathrm{dl}$, arterial $\mathrm{pH}$ of $\leq 7.3$, bicarbonate level of $\leq 18 \mathrm{mEq} / \mathrm{L}$, and adjusted for albumin anion gap of $>10-12$. Positive serum and urine ketones may further support the diagnosis of DKA (Gosmanov et al., 2014). In our practice at Elobied hospital, the diagnosis of DKA depends on the presenting symptoms, hyperglycaemia, and the presence of glucose and acetone in the urine. The reason for incomplete investigations is due to shortage of laboratory facilities. Mean blood glucose level in patients with DKA was $368 \pm 72 \mathrm{mg} / \mathrm{dl}$.

Although persons with diabetic ketoacidosis typically have a history of diabetes mellitus, in this study $32 \%$ of patients have newly diagnosed diabetes. Similar results were reported in study from USA (Westerberg, 2013).

Traditionally, the severity of DKA is determined by the arterial $\mathrm{pH}$, bicarbonate level, anion gap, and mental status of the patient (Westerberg 2013). In our practice at Elobied hospital, we depend on mental status of the patient, pulse, and the systolic blood pressure. In this study $14(26 \%)$ patients were diagnosed as having severe DKA due to presence of systolic hypotension (systolic blood pressure $<90 \mathrm{mmHg}$ ) and/or tachycardia (Heart rate $>100$ beat/minute). All patients with severe DKA belonged to elder age group ( $>55$ years). It is important to assess the severity of DKA to determine the clinical setting in which the patient is to be managed (Noble-Bell and Cox, 2014). Unfortunately there is no intensive care unit (ICU) in Elobied hospital, and therefore patients with DKA are managed in the general medical wards in regard to the degree of their illness.

In this study all patients admitted DKA were managed by giving soluble insulin for hyperglycaemia and $0.9 \%$ $\mathrm{NaCl}$ for correction of fluid deficit beside treatment of infections if present. Records showed no evidence of bicarbonate or potassium administration. This approach of management of DKA is similar to what had been mentioned by Trachtenbarg (2005), that intravenous insulin and fluid replacement are the mainstay of therapy with careful monitoring of potassium levels.

In spite of limited resources and facilities in our hospital, in this study the overall in-hospital mortality rate was $3.8 \%$, which was much lower than mortality rate in study from Syria (Alourfi et al., 2015). Similar mortality rate was reported by Barski et al. (2012). In this study the death was attributed to severe comorbid diseases like acute kidney injury rather to metabolic complications of DKA. This important comment about the cause of death was also mentioned by Barski et al. (2012), in their study.

\section{Conclusion}

Since the majority of cases of DKA occurs in patients who are known diabetics, this complication should be largely preventable by education of patients, health care professionals and the general public. Laboratory facilities at Elobied hospital must be improved to encourage early proper diagnosis of DKA and determine the severity of DKA to decide where the patient can be managed, in the intensive care unit or in the general medical wards. Intensive care unit (ICU) must be established to provide good care for severe DKA and others emergency cases.

\section{Conflict of interest statement}

Authors declare that they have no conflict of interest.

\section{References}

Alourfi, Z., Homsi, H., 2015. Precipitating factors, outcomes, and recurrence of diabetic ketoacidosis at a university hospital in Damascus. Avicenna J. Med. 5(1), 11-15. 
Barski, L., Nevzorov, V., Rabaaev, E., Jotkawitz, A., HarmanBoehm, I., Zektser, M., Zeller, L., Shleyter, E., Almong, Y., 2012. Diabetic ketoacidosis: precipitating factors and outcomes of care. Isr. Med. Assoc. J. 14, 299-303.

Gosmanov, A.R., Gosmanov, E.O., Dillard-Cannon, E., 2014. Management of adult diabetic ketoacidosis. Diabetes Metab. Syndr. Obes. 7, 255-264.

Maletkovic, J., Drexler, A., 2013. Diabetic ketoacidosis and hyperglycaemic hyperosmolar state. Endocrinol. Metab. Clin. North Am. 42(4), 677-695.

Noble-Bell, G., Cox, A., 2014. Management of biabetic ketoacidosis in adults. Nurs. Times. 110(10), 14-17.
Nugent, B. W., 2005. Hyperosmolar hyperglycemic state. Emerg. Med. Clin. North Am. 23(3), 629-648.

Trachtenbarg, D.E., 2005. Diabetic ketoacidosis. Am. Fam. Physician. 71(9), 1705-1714.

Umpierrez, G. E., Murphy, M. B., Kitabchi, A. E., 2002. Diabetic ketoacidosis and hyperglycemic hyperosmolar syndrome. Diabetes Spectr. 15(1), 28-36.

Umpierrez, G.E., Kitabchi, A.E., 2003. Diabetic ketoacidosis: risk factors and management strategies. Treat. Endocrinol. 2(2), 95-108.

Westerberg, D.P., 2013. Diabetic Ketoacidosis: evaluation and treatment. Am. Fam. Physician. 87(5), 337-346.

\section{How to cite this article:}

Hamad Elfaki, A. M., Agab, M. A., 22016. Adult diabetic ketoacidosis: Precipitating factors, diagnostic criteria and management strategies at Elobied Hospital - West Sudan. Int. J. Curr. Res. Biosci. Plant Biol. 3(8), 65-68. doi: http://dx.doi.org/10.20546/ijcrbp.2016.308.010 